\title{
The treatment of early rectal cancer in the era of adjuvant and neo-adjuvant therapy
}

\author{
Michael G. Thomas, David E. Messenger, Katherine Gash \\ Department of Colorectal Surgery, University Hospitals Bristol NHS Foundation Trust, Bristol BS2 8HW, UK.
}

Correspondence to: Dr. Michael G. Thomas, Department of Colorectal Surgery, Bristol Royal Infirmary, Marlborough Street, Bristol BS2 8HW, UK. E-mail: Michael.Thomas@UHBristol.nhs.uk

\begin{abstract}
How to cite this article: Thomas MG, Messenger DE, Gash K. The treatment of early rectal cancer in the era of adjuvant and neo-adjuvant therapy. Mini-invasive Surg 2018;2:17. http://dx.doi.org/10.20517/2574-1225.2018.25
\end{abstract}

Received: 9 May 2018 First Decision: 15 Jun 2018 Revised: 22 Jun 2018 Accepted: 2 Jul 2018 Published: 18 Jul 2018

Science Editor: Gordon Buchanan Copy Editor: Jun-Yao Li Production Editor: Huan-Liang Wu

\begin{abstract}
The accurate staging of rectal cancer improves the stratification of patients for adjuvant therapy. Staging of tumor with endoluminal ultrasonography (EUS) shows a good correlation with histology $(\kappa=0.85 ; 95 \%$ confidence interval 0.76 0.95). Overall pT and pN stage accuracy of EUS was $92 \%$ and $65 \%$ respectively. The staging of local disease can be further augmented by EUS guided fine needle aspiration of extra rectal lesions lying within or outside of the mesorectum. In a systematic review of local excision after neoadjuvant therapy a total of 22 unique studies reporting on 1068 patients were analysed. At a median follow-up of 54 months, ypTO tumours had a pooled local recurrence rate of $4 \%$ and a median disease-free survival rate of $95 \%$. Outcomes for $\geq$ ypT1 tumours were much worse with pooled local recurrence and disease-free survival of $22 \%$ and $68 \%$, respectively. In a review of 22 studies, 804 patients who underwent local excision followed by adjuvant therapy either for unfavourable histology, prohibitive comorbidity or patient choice. the pooled local recurrence was $5.8 \%$ for pT1 tumours, $13.8 \%$ for pT2 tumours and $33.7 \%$ for pT3 tumours. In addition, the response to radiotherapy may be enhanced by aspirin, metformin and statins.
\end{abstract}

Keywords: Early rectal cancer, local excision, neoadjuvant chemoradiotherapy

\section{BACKGROUND}

The treatment of rectal cancer has advanced considerably during the last 30 years. It is widely accepted that surgery should be based on sound oncological principles where the aim is to completely excise the surrounding mesorectum in order to achieve a resection margin free from microscopic disease, together with an adequate lymph node harvest. Commonly referred to as total mesorectal excision (TME), this technique in combination with accurate post-operative staging has improved the oncological results of

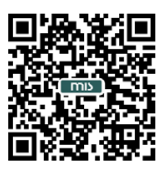


surgery ${ }^{[1-3]}$. Nevertheless, the debate with regards to the necessity to completely remove the mesorectum in the setting of early rectal cancer is still not fully resolved. What is clear from the evidence is that involvement of the circumferential resection margin (CRM) remains one of the most powerful predictors of local recurrence in rectal cancer.

The Swedish Rectal Cancer and Dutch TME trials gave credence to the concept that local control could be enhanced by the addition of neoadjuvant short course radiotherapy (SCRT) ${ }^{[3,4]}$. Indeed, the National Institute of Clinical Excellence in the United Kingdom currently recommends that neoadjuvant treatment is administered in the form of SCRT for moderate-risk tumours (cT3b or greater or suspected nodal involvement or venous invasion) and as long course chemoradiotherapy (LCCRT) for high-risk tumours that either threaten the circumferential resection margin or encroach on the levator plate ${ }^{[5]}$. Radical surgery remains the cornerstone of treatment for locally advanced rectal cancer with 5-year local recurrence (LR) and disease-free survival rates of $4 \%$ and $86 \%$, respectively ${ }^{[1,2]}$, and a 30 -day mortality rate of $0.9 \%-1.5 \%{ }^{[4,6-8]}$. The down side of radical surgery is that, even in experienced hands, morbidity occurs in $38 \%-54 \%$ of patients $^{[4,6-8]}$ and is associated with a significant adverse impact on quality of life with elevated levels of bowel, urinary, and sexual dysfunction ${ }^{[9-12]}$. In addition, there is a perception in the UK that local control is enhanced in disease that threatens the mesorectal rectal fascia with the use of LCCRT. Moreover, the use of LCCRT may result in a pathological complete response (pCR) in $15 \%$ to $25 \%$ of cases with contemporary neoadjuvant LCCRT regimens ${ }^{[13-15]}$. This has led to the concept of watchful waiting, the success of which is clearly dependent on the accurate prediction of a complete clinical response (cCR) and does not necessarily correspond to a pCR. Of note, in a pooled analysis of neoadjuvant treatment studies, cCR was associated with pCR in only $30 \%$ of patients ${ }^{[16]}$. It is noteworthy, however, that a PCR is heavily dependent on the quality of the surgical specimen received and the accuracy of pathological examination, which may vary considerably $^{[17,18]}$.

In view of the downstaging effect of LCCRT and the potential to obtain a pCR, there is increasing evidence to suggest that patients with pCR could be safely managed by local excision. Local procedures, such as transanal endoscopic microsurgery (TEM), which was first described in the 1980s, and more recently, transanal endoscopic operation (TEO) and transanal minimally invasive surgery (TAMIS), can potentially avoid the morbidity associated with radical surgery and enable organ preservation ${ }^{[19]}$. A recent systematic review by our own group suggested that local excision after neoadjuvant therapy for rectal cancer should only be considered as curative, with an acceptable level of local control, if a pCR was obtained. Pooled local recurrence rates were significantly greater and median disease-free survival significantly lower among tumours staged as ypT1 or above compared to ypTo tumours ${ }^{[2]}$. In this article we describe our approach to the management of early rectal cancer, its staging and our evidence-based rationale for the use of neoadjuvant and adjuvant therapies.

\section{STAGING EARLY RECTAL CANCER}

The accurate staging of colorectal neoplasia can improve the stratification of patients for adjuvant treatment. We strongly believe that endoluminal ultrasound is a powerful tool in staging early disease. In support of this view, our initial work focussed on predicting the mural extent of neoplasia. Colonoscopic endoluminal ultrasonography (EUS) was used in a prospective study to determine the stage of rectosigmoid neoplasia in 121 patients. Mural tumour (T) stage was designated by EUS as uTo/1-uT4 in 121 patients. Specific nodal (N) staging was performed in 39 of these cases. EUS staging was compared with histological stage $(\mathrm{pT}$ and $\mathrm{pN})$ in 93 patients who underwent resection. Mural staging of disease using colonoscopic EUS showed good correlation with histopathological stage ( $\kappa=0.85 ; 95 \%$ confidence interval 0.76-0.95). Overall $\mathrm{pT}$ and $\mathrm{pN}$ stage accuracy of EUS was $92 \%$ and $65 \%$ respectively ${ }^{[21]}$. 
In a later study, the accuracy of colonoscopic EUS was assessed in the selection of patients with rectal neoplasia suitable for local excision by TEM. Patients with premalignant (uTo) lesions or with uT1 tumours that had favourable histology were offered a TEM. This has been our preferred method of local excision since 1996. Data were collected prospectively over a six-year period. The preoperative stage predicted by EUS (uT stage) was compared to the postoperative histopathological stage of the resected specimens (pT stage). One hundred and fifty-six EUS examinations were evaluated. Sixty-two patients went on to have TEM whilst the remaining 94 had another form of surgery. Of the 62 patients undergoing TEM, 3 were over staged on EUS. No patients were understaged, giving an accuracy of $95 \%$. The accuracy of EUS at predicting more advanced disease fell to $89 \%$, giving an overall accuracy of $92 \%^{[22]}$. Indeed, we feel that EUS in our institution is highly accurate at predicting To/1 vs. T2 disease and we routinely use EUS in combination with CT and MRI before planning intervention. In addition, the staging of local disease can be further augmented by colonoscopic EUS guided fine needle aspiration of extra rectal lesions lying within or outside of the mesorectum that are demonstrated on cross-sectional imaging ${ }^{[23]}$.

The need for highly accurate staging for early rectal cancer and early stage disease is highlighted by the observation that the National Bowel Cancer Screening Programme in the United Kingdom will result in an increase in the proportion of early stage tumours that are potentially amenable to local excision, although stage migration has yet to be demonstrated in population-based studies. Local recurrence rates of $<5 \%$ after TEM excision have been reported for pT1 tumours with favourable histology that equate to those achieved by radical surgery. As local excision offers the possibility of organ preservation and thus improved quality of life, recent focus has therefore shifted toward the use of neoadjuvant therapy as a means of downstaging early tumours (cT1-T3a) and sterilizing the mesorectal nodal field before local excision.

\section{TREATMENT STRATEGY}

Given the reluctance to administer neoadjuvant radiotherapy in early rectal cancer, there is a lack of data addressing the oncological outcomes and morbidity profile of this approach. This prompted us to undertake our systematic review of LE after neoadjuvant therapy to determine oncological outcomes as defined by LR and, second, to determine the incidence and nature of postoperative complications ${ }^{[20]}$. A total of 22 unique studies reporting on 1068 patients were analysed. Pre-treatment T2 and T3 tumours accounted for $46.4 \%$ and $30.7 \%$ of cases, respectively and LCCRT was administered in all studies, except to a cohort of 64 patients who received SCRT. The pooled CCR based on the staging modalities used in these studies was $45.8 \%$ with a pooled pCR $44.2 \%$. At a median follow-up of 54 months, ypTo tumours had a pooled local recurrence rate of $4 \%$ and a median disease-free survival rate of $95 \%$. This compared favourably to results achieved with radical surgery in equivalent stage disease. Outcomes for $\geq y p T 1$ tumours were much worse with pooled local recurrence and disease-free survival of $22 \%$ and $68 \%$, respectively ${ }^{[20]}$. Despite the obvious limitations of study heterogeneity and their retrospective nature, we conclude that local excision should only be considered as a definitive therapy if a pCR, i.e., ypTo, is obtained in the excision specimen.

The other unresolved issue is whether radiotherapy should be used in an adjuvant setting to improve outcomes after local excision of early rectal cancer, specifically in pT1 disease with unfavourable histology and $\mathrm{pT} 2 / \mathrm{pT} 3 \mathrm{a}$ disease. It is our current practice to offer local excision alone to patients with pT1 disease with favourable histology. Where post-operative histology reveals pT1 disease with adverse histological features or unexpected $\mathrm{pT} 2$ disease, we offer completion radical surgery as the standard of care and reserve adjuvant LCCRT for patients either deemed unfit or who wish to preserve their rectum. Even though much of the recent work in this field has focussed on the use of neoadjuvant therapy prior to local excision, we conducted our own systematic review of local excision followed by adjuvant therapy to determine if this was an acceptable treatment option. In this review, 22 studies described 804 patients who underwent local excision followed by adjuvant therapy either for unfavourable histology, prohibitive comorbidity or 
patient choice. T1, T2 and T3 tumours accounted for 35.1\%, 58.0\% and 6.9\% of cases, respectively. Adjuvant regimens were exclusively long course in nature and included radiotherapy either alone or combination with chemotherapy. At a median follow-up of 51 months, the pooled local recurrence was $5.8 \%$ for pT1 tumours, $13.8 \%$ for $\mathrm{pT} 2$ tumours and $33.7 \%$ for $\mathrm{pT} 3$ tumours ${ }^{[2]}$. This suggests that it is possible to achieve reasonable local control in $\mathrm{pT} 1$ disease, even if adverse histological features are present, with post-operative adjuvant therapy. Clearly, this treatment strategy must be offered in the context of the significant body of evidence for radical surgery and the clinician most weigh up the balance of organ preservation, quality of life, patient choice and oncological outcome on an individual patient basis.

\section{AUGMENTING THE RESPONSE TO RADIOTHERAPY}

Tumour regression following neo-adjuvant chemo-radiotherapy is significantly associated with 5 -year overall survival, disease-free survival and local recurrence ${ }^{[25,26]}$, thus increasing our understanding of treatment adjuncts that might enhance tumour response to therapy. Also, adjuncts might increase the likelihood of pCR or near-pCR in patients with rectal cancer. A systematic literature search was conducted and all studies investigating the use of drugs to enhance response to neoadjuvant radiation in rectal cancer. However, the small number of studies and the heterogeneity of outcomes precluded systematic review and meta-analysis from being undertaken ${ }^{[27]}$. We therefore outlined the evidence to date in a narrative review and explored the potential mechanisms of action. Despite the obvious limitations, aspirin, metformin and statins appear to be associated with down staging rectal tumours and thus could potentially play the role of adjuncts in neoadjuvant therapy ${ }^{[28-31]}$. Moreover, provisional research strongly supports the potential role for the use of aspirin to induce apoptosis and enhance the effect of pre-operative radiotherapy ${ }^{[31]}$. This has prompted us to conduct a proof of principle prospective cohort study comparing patients who are taking aspirin to those who do not whilst receiving neo-adjuvant chemoradiotherapy prior to laparoscopic and open resection for rectal cancer (the ASPIRE study). The end points for this proof of principle study are both traditional surrogate markers of oncological outcome and laboratory markers of response to chemoradiotherapy.

\section{FOLLOW UP IN PATIENTS WITH AN APPARENT COMPLETE CLINICAL RESPONSE}

Around $15 \%-25 \%$ of tumours have a $\mathrm{PCR}^{[32,33]}$, which is associated with a reduction in local recurrence and improved disease-free and overall survival ${ }^{[3,35]}$. Despite the difficulties in predicting pCR based on clinical and radiological findings, there appears to be increasing evidence that patients who exhibit an apparent cCR could be safely managed by local procedures, such as TEMS, TEO or TAMIS, to the tumour site or indeed intensive surveillance, the so called "watch and wait" strategy, to avoid the morbidity associated with radical surgery and enable organ preservation ${ }^{[36-38]}$. There is a paucity of evidence on the optimum follow up of such patients and we have therefore erred on the side of caution with intensive follow up for in the first 2 years that consists of endoscopic evaluation \pm mucosal biopsy, MRI, EUS and CT every 3 months. The surveillance interval is then extended to 6 months, thereafter. Large prospective cohort studies will be required to educate this debate.

\section{CONCLUSION}

We provide treatment for early rectal cancer that is both patient-centred and based on the available evidence. This follows a detailed staging strategy that consists of clinical examination, CT, MRI, PET and the use of colonoscopic EUS with or without fine needle aspiration of extramural pelvic disease. All cases are discussed at a weekly multidisciplinary meeting where the patient's comorbidities, quality of life and preferences are discussed in conjunction with the likely oncological outcomes of the potential treatment regimens on offer. Consent is usually a two or three-staged process where the patient's treatment options are discussed in detail. Rather than adopting a protocol-driven strategy, we are strong advocates 
of using an evidence-based strategy that is tailored to the individual patient. In patients in whom we can confidently predict favourable T1 disease we would offer local excision, either by TEM or standard transanal excision as appropriate, or radical resection. In patients who opt for local excision and have pT1 disease with unfavourable histology, then we would offer adjuvant LCCRT in addition to standard radical resection. For $\mathrm{pT} 2$ disease and above, oncological options are compromised, and we would strongly recommend completion radical surgery. In patients with cT1/T2 disease that appears amenable to local excision, we offer the options of neoadjuvant therapy prior to LE or conventional resection. If patients elect to undergo neoadjuvant therapy and local excision, then we undertake a detailed assessment of treatment response using endoscopy, colonoscopic EUS and MRI prior to surgery. If a CCR is demonstrated, then we will operate a watchful waiting policy in selected cases. Where a significant partial response or nearcCR has been obtained, then we would proceed to local excision. This also gives us the option to offer completion radical surgery if there has been minimal tumour regression or the disease has progressed despite neoadjuvant treatment.

While the STAR-TREC trial aims to compare differing neoadjuvant regimens with radical surgery in early rectal cancer $(\mathrm{T} 1-\mathrm{T} 3 \mathrm{a})^{[38]}$, a direct comparison of neoadjuvant and local excision versus local excision and adjuvant therapy has never been compared in a prospective study. It is possible that this debate may never been resolved with randomized controlled trials owing to the complexity in study design. It is likely that the neoadjuvant $v s$. adjuvant debate may only be answered with the use of large scale prospective registries. The management of early rectal cancer that combines local excision techniques with neoajuvant/adjuvant therapy is an evolving area of practice and we await the results of future studies with interest.

In patients with pT2 disease with unfavourable histology or pT3 disease not breaching the mesorectal resection margin (based on MRI) we offer conventional laparoscopic or open anterior resection with or without short course radiotherapy. In patients with more advanced disease our preference is to routinely offer pre-operative CRT followed by surgery in 3-6 months. Indeed, we are moving towards at least 3 months following CRT after post-operative re staging. In patients with low disease not suitable for anterior resection or local excision we favour extralevator abdominoperineal excision with immediate biologic mesh reconstruction of the pelvic inlet augmented by the use of myocutaneous flaps where indicated ${ }^{[39,40]}$. Our use of myocutaneous flaps has evolved over the last 10 years and we have recently found that bilateral gluteal advancement flaps provide excellent healing and quality of life (unpublished data, Thomas, Warr, Longman, Messenger).

\section{DECLARATIONS}

\section{Authors' contributions}

Clinical and scientific data: Thomas MG, Messenger DE, Gash K

Systematic review data: Thomas MG, Messenger DE

Manuscript preparation: Thomas MG, Messenger DE, Gash K

\section{Availability of data and materials}

Not applicable.

\section{Financial support and sponsorship}

The study was supported by Fulbright Scholarship, Above and Beyond Research Trust, David Telling Trust, RCS research Scholership, Bristol Cancer Research UK 5 year programme grant, and John James Foundation Bristol.

\section{Conflicts of interest}

All authors declare that there are no conflicts of interest. 


\section{Ethical approval and consent to participate}

Not applicable.

\section{Consent for publication}

Not applicable.

\section{Copyright}

(c) The Author(s) 2018.

\section{REFERENCES}

1. Aitken RJ. Mesorectal excision for rectal cancer. Br J Surg 1996;83:214-6.

2. Enker WE, Thaler HT, Cranor ML, Polyak T. Total mesorectal excision in the operative treatment of carcinoma of the rectum. J Am Coll Surg 1995;181:335-46.

3. van Gijn W, Marijnen CA, Nagtegaal ID, Kranenbarg EM, Putter H, Wiggers T, Rutten HJ, Påhlman L, Glimelius B, van de Velde CJ; Dutch Colorectal Cancer Group. Preoperative radiotherapy combined with total mesorectal excision for resectable rectal cancer: 12-year follow up of the multicentre, randomised controlled TME trial. Lancet Oncol 2011;12:575-82.

4. Pettersson D, Cedermark B, Holm T, Radu C, Påhlman L, Glimelius B, Martling A. Interim analysis of the Stockholm III trial of preoperative radiotherapy regimens for rectal cancer. Br J Surg 2010;97:580-7.

5. National Collaborating Centre for Cancer. Colorectal Cancer: The Diagnosis and management of Colorectal Cancer. Cardiff, United Kingdom: National Collaborating Centre for Cancer; 2011.

6. Penninckx F, Kartheuser A, Van de Stadt J, Pattyn P, Mansvelt B, Bertrand C, Van Eycken E, Jegou D, Fieuws S; PROCARE. Outcome following laparoscopic and open total mesorectal excision for rectal cancer. Br J Surg 2013;100:1368-75.

7. van der Pas MH, Haglind E, Cuesta MA, Fürst A, Lacy AM, Hop WC, Bonjer HJ; COlorectal cancer Laparoscopic or Open Resection II (COLOR II) Study Group. Laparoscopic versus open surgery for rectal cancer (COLOR II): short-term outcomes of a randomised, phase 3 trial. Lancet Oncol 2013;14:210-8.

8. Fleshman J, Branda M, Sargent DJ, Boller AM, George V, Abbas M, Peters WR Jr, Maun D, Chang G, Herline A, Fichera A, Mutch M, Wexner S, Whiteford M, Marks J, Birnbaum E, Margolin D, Larson D, Marcello P, Posner M, Read T, Monson J, Wren SM, Pisters PW, Nelson H. Effect of laparoscopic assisted resection vs open resection of stage II or III rectal cancer on pathologic outcomes: the ACOSOG Z6051 randomized clinical trial. JAMA 2015;314:1346-55

9. Hendren SK, O'Connor BI, Liu M, Asano T, Cohen Z, Swallow CJ, Macrae HM, Gryfe R, McLeod RS. Prevalence of male and female sexual dysfunction is high following surgery for rectal cancer. Ann Surg 2005;242:212-23.

10. Engel J, Kerr J, Schlesinger-Raab A, Eckel R, Sauer H, Hölzel D. Quality of life in rectal cancer patients: a four-year prospective study. Ann Surg 2003;238:203-13.

11. Wilson TR, Alexander DJ. Clinical and non-clinical factors influencing postoperative health-related quality of life in patients with colorectal cancer. Br J Surg 2008;95:1408-15.

12. Wallner C, Lange MM, Bonsing BA, Maas CP, Wallace CN, Dabhoiwala NF, Rutten HJ, Lamers WH, Deruiter MC, van de Velde CJ; Cooperative Clinical Investigators of the Dutch Total Mesorectal Excision Trial. Causes of fecal and urinary incontinence after total mesorectal excision for rectal cancer based on cadaveric surgery: a study from the Cooperative Clinical Investigators of the Dutch Total Mesorectal Excision Trial. J Clin Oncol 2008;26:4466-72.

13. Maas M, Nelemans PJ, Valentini V, Das P, Rödel C, Kuo LJ, Calvo FA, García-Aguilar J, Glynne-Jones R, Haustermans K, Mohiuddin M, Pucciarelli S, Small W Jr, Suárez J, Theodoropoulos G, Biondo S, Beets-Tan RG, Beets GL. Long-term outcome in patients with a pathological complete response after chemoradiation for rectal cancer: a pooled analysis of individual patient data. Lancet Oncol 2010;11:835-44

14. de Campos-Lobato LF, Stocchi L, da Luz Moreira A, Geisler D, Dietz DW, Lavery IC, Fazio VW, Kalady MF. Pathologic complete response after neoadjuvant treatment for rectal cancer decreases distant recurrence and could eradicate local recurrence. Ann Surg Oncol 2011;18:1590-8.

15. Garcia-Aguilar J, Smith DD, Avila K, Bergsland EK, Chu P, Krieg RM; Timing of Rectal Cancer Response to Chemoradiation Consortium. Optimal timing of surgery after chemoradiation for advanced rectal cancer: preliminary results of a multicenter, nonrandomized phase ii prospective trial. Ann Surg 2011;254:97-102.

16. Hiotis SP, Weber SM, Cohen AM, Minsky BD, Paty PB, Guillem JG, Wagman R, Saltz LB, Wong WD. assessing the predictive value of clinical complete response to neoadjuvant therapy for rectal cancer: an analysis of 488 patients. J Am Coll Surg 2002;194:131-5.

17. Chua YJ. Pathological complete response: still a relevant endpoint in rectal cancer? Lancet Oncol 2010;11:807-8.

18. Habr-Gama A, Perez R, Proscurshim I, Gama-Rodrigues J. Complete clinical response after neoadjuvant chemoradiation for distal rectal cancer. Surg Oncol Clin N Am 2010;19:829-45.

19. Bach SP, Hill J, Monson JR, Simson JN, Lane L, Merrie A, Warren B, Mortensen NJ; Association of Coloproctology of Great Britain and Ireland Transanal Endoscopic Microsurgery (TEM) Collaboration. Transanal endoscopic microsurgery (TEM) Collaboration. a predictive model for local recurrence after transanal endoscopic microsurgery for rectal cancer. Br J Surg 2009;96:280-90.

20. Hallam S, Messenger D, Thomas M. A systematic review of local excision after neoadjuvant therapy for rectal cancer: are ypT0 tumors the limit? Dis Colon Rectum 2016;59:984-97.

21. Norton SA, Thomas MG. Staging of rectosigmoid neoplasia with colonoscopic endoluminal ultrasonography. Br J Surgery 1999;86:9426. 
22. Glancey D, Pullyblank A, Thomas M. The role of colonoscopic endoanal ultrasound scanning (EUS) in selecting patients suitable for resection by transanal endoscopic microsurgery (TEM). Colorectal Dis 2005;7:148-50.

23. Lim J, Norton SA, Wong NA, Thomas MG. Endoscopic ultrasound guided fine needle aspiration of extra-rectal lesions. Tech Coloproctol 2017;21:393-5.

24. Cuttin J, Hallam S, Thomas M, Messenger D. A systematic review of local excision followed by adjuvant therapy in early rectal cancer: are pT1 tumours the limit? Colorectal Dis 2018; accepted for publication.

25. Swedish Rectal Cancer Trial; Cedermark B, Dahlberg M, Glimelius B, Påhlman L, Rutqvist LE, Wilking N. Improved survival with preoperative radiotherapy in resectable rectal cancer. .N Engl J Med 1997;336:980-7.

26. Kapiteijn E, Marijnen CA, Nagtegaal ID, Putter H, Steup WH, Wiggers T, Rutten HJ, Pahlman L, Glimelius B, van Krieken JH, Leer JW, van de Velde CJ; Dutch Colorectal Cancer Group. Preoperative radiotherapy combined with total mesorectal excision for resectable rectal cancer. N Engl J Med 2001;345:638-46.

27. Sauer R, Becker H, Hohenberger W, Rodel C, Wittekind C, Fietkau R, Martus P, Tschmelitsch J, Hager E, Hess C, Karstens J, Liersch T, Schmidberger H, Raab R. Preoperative versus postoperative chemoradiotherapy for rectal cancer. N Engl J Med 2004;351:1731-40.

28. Gash K, Chambers A, Cotton D, Williams AC, Thomas MG. Potentiating the effects of radiotherapy in rectal cancer: the role of aspirin, statins and metformin as adjuncts to therapy. Br J Cancer 2017;117:210-9.

29. Restivo A, Cocco IMF, Casula G, Scintu F, Cabras F, Scartozzi M, Zorcolo L. Aspirin as a neoadjuvant agent during preoperative chemoradiation for rectal cancer. Br J Cancer 2015;113:1133-9.

30. Rothwell PM, Wilson M, Elwin C, Norrving B, Algra A, Warlow CP, Meade TW. Long-term effect of aspirin on colorectal cancer incidence and mortality: 20-year follow-up of five randomised trials. Lancet 2010;376:1741-50.

31. Gash K, Collard T, Chambers A, Paraskevia A, Williams A, Thomas M. Aspirin enhances the response to radiation in colorectal cancer cell lines. Gut 2015;64 Suppl 1:A533.

32. Mace AG, Pai RK, Stocchi L, Kalady MF. American Joint Committee on Cancer and College of American Pathologists regression grade: a new prognostic factor in rectal cancer. Dis Colon Rectum 2015;58:32-44.

33. Rödel C, Martus P, Papadoupolos T, Füzesi L, Klimpfinger M, Fietkau R, Liersch T, Hohenberger W, Raab R, Sauer R, Wittekind C. Prognostic significance of tumor regression after preoperative chemoradiotherapy for rectal cancer. J Clin Oncol 2005;23:8688-96.

34. Roh MS, Colangelo LH, O’Connell MJ, Yothers G, Deutsch M, Allegra CJ, Kahlenberg MS, Baez-Diaz L, Ursiny CS, Petrelli NJ, Wolmark N. Preoperative multimodality therapy improves disease-free survival in patients with carcinoma of the rectum: NSABP R-03. J Clin Oncol 2009;27:5124-30.

35. Habr-Gama A, Sabbaga J, Gama-Rodrigues J, Sao Juliao GP, Proscurshim I, Bailao Aguilar P, Nadalin W, Perez RO. Watch and wait approach following extended neoadjuvant chemoradiation for distal rectal cancer: are we getting closer to anal cancer management? Dis Colon Rectum 2013;56:1109-17.

36. Ryan JE, Warrier SK, Lynch AC, Ramsay RG, Phillips WA, Heriot AG. Predicting pathological complete response to neoadjuvant chemoradiotherapy in locally advanced rectal cancer: a systematic review. Colorectal Dis 2016;18:234-46.

37. Martin ST, Heneghan HM, Winter DC. Systematic review and meta-analysis of outcomes following pathological complete response to neoadjuvant chemoradiotherapy for rectal cancer. Br J Surg 2012;99:918-28.

38. University of Birmingham. Can the Rectum be Saved by Watchful Waiting or TransAnal Surgery Following (Chemo)Radiotherapy Versus Total Mesorectal Excision for Early REctal Cancer? (STAR-TREC). ClinicalTrials.gov Identifier: NCT02945566.

39. Foster JD, Pathak S, Smart NJ, Branagan G, Longman RJ, Thomas MG, Francis N. Reconstruction of the perineum following extralevator abdomino-perineal excision for carcinoma of the lower rectum - a systematic review. Colorectal Dis 2012;14:1052-9.

40. Smart N, Thomas M, Longman R. Perineal reconstruction after abdominoperineal excision using inferior gluteal artery perforator flaps (Br J Surg 2012; 99: 584-588). Br J Surg 2012;99:1165-6; author reply 1166. 